\title{
ON DIFFERENTIAL EQUATIONS OF VON GEHLEN AND ROAN
}

\author{
ETSURO DATE \\ Department of Pure and Applied Mathematics, Graduate School of Information \\ Science and Technology, Osaka University \\ e-mail:date@ist.osaka-u.ac.jp
}

\begin{abstract}
Polynomials appearing in the description of ground states of superintegrable chiral Potts models are shown to satisfy a special class of generalised hypergeometric differential equations after a simple modification. This proves a conjecture of von-Gehlen and Roan.
\end{abstract}

2000 Mathematics Subject Classification. 47E05, 82B23

1. Introduction. Let $N \geq 2$ be a positive integer and $\omega=\exp (2 \pi i / N)$ a primitive $N$ th root of unity. Take a pair of linear operators $X, Z \in \operatorname{End}\left(\mathbf{C}^{N}\right)$ that satisfies the following commutation relation and the normalisation condition:

$$
Z X=\omega X Z, \quad X^{N}=Z^{N}=i d
$$

The superintegrable chiral Potts Hamiltonian (see for example [1], [2]) on a chain of length $L$ is a linear operator on $\left(\mathbf{C}^{N}\right)^{\otimes L}$ defined by

$$
H\left(k^{\prime}\right)=-\sum_{l=1}^{L} \sum_{n=1}^{N-1} \frac{2}{1-\omega^{-n}}\left(X_{l}^{n}+k^{\prime} Z_{l}^{n} Z_{l+1}^{N-n}\right),
$$

where $k^{\prime}$ is a real parameter and $X_{l}$ denotes the operator acting on the $l$ th component as $X$ and for other components as identity.

Note that if we write

$$
H\left(k^{\prime}\right)=H_{0}+k^{\prime} H_{1}
$$

$H_{0}$ and $H_{1}$ satisfy the Dolan-Grady relation

$$
\left[H_{i},\left[H_{i},\left[H_{i}, H_{j}\right]\right]\right]=4 N^{2}\left[H_{i}, H_{j}\right], \quad i, j=0,1
$$

and give a representation of the so-called Onsager algebra, which can also be viewed as either a deformation of the nilpotent part of the affine Lie algebra of type $A_{1}^{(1)}$ or a quotient of the loop algebra of $\mathfrak{s l}_{2}$.

The principal problem in statistical mechanics defined by this operator is to find eigenvalues and eigenvectors. Bethe Ansatz affords us a method for such purpose. 
It is known that ground state eigenvalues and eigenvectors are described by zeroes of polynomials $F_{j}$ (cf. $\left.[\mathbf{1}, \mathbf{2}, \mathbf{3}, \mathbf{4}, \mathbf{6}]\right)$ defined by the relation

$$
\left(\frac{t^{N}-1}{t-1}\right)^{L}=\sum_{j=0}^{N-1} t^{j} F_{j+1}(s), \quad s=t^{N} .
$$

In $[3,4,6]$ von Gehlen and Roan derived a system of first-order differential equation for $F_{j}$.

The vector of polynomials

$$
F={ }^{t}\left(F_{1}, F_{2}, \ldots, F_{N}\right)
$$

satisfy

$$
\begin{gathered}
N s(s-1) \frac{d F}{d s}=B F, \\
B=\left(\begin{array}{cccc}
d_{0} & -L s & \ldots & -L s \\
-L & d_{1} & \ddots & \vdots \\
\vdots & \ddots & \ddots & -L s \\
-L & \cdots & -L & d_{N-1}
\end{array}\right), \\
d_{j}=L(N-1) s-j(s-1) .
\end{gathered}
$$

When $N=2$ (the Ising case) each polynomial satisfies Gauß hypergeometric differential equation. Further by a suitable change of variable the polynomials turn out to be Chebyshev polynomials, and this was convenient for the description of eigenvalues.

Proceeding further they also derived third-order differential equations for the case $N=3$. One of them takes the following form:

$$
\begin{aligned}
& 27 s^{2}(s-1)^{2} F_{1}^{\prime \prime \prime}-27 s(s-1)((2 L-4) s+2) F_{1}^{\prime \prime} \\
& \quad+3\left(3 L^{2} s(4 s-1)-3 L s(10 s-7)+2(s-1)(10 s-1)\right) F_{1}^{\prime} \\
& \quad-(L-1)\left(L(L(8 s+1)-4(s-1)) F_{1}=0 .\right.
\end{aligned}
$$

These equations have regular singular points only at $s=0,1, \infty$, although this in not explicitly mentioned in $[3,4,6]$. They also studied the zeroes of polynomials in the case of $N=3$ numerically.

Based on such calculations they conjectured that each of $F_{j}$ satisfies an $N$ th-order ordinary differential equations of the form that follows.

Conjecture 1.

$$
\begin{aligned}
& N^{N} s^{N-1}(s-1)^{N-1} \frac{d^{N} F_{j}}{d s^{N}} \\
& +\sum_{k=1}^{N-1} N^{k} s^{k-1}(s-1)^{k-1} D_{j k}(s) \frac{d^{k} F_{j}}{d s^{k}}+D_{j 0}(s) F_{j}=0,
\end{aligned}
$$

where $D_{j k}$ are polynomials in $s$.

In this paper we show that after a simple transformation the scalar differential equations in question are generalised hypergeometric differential equations, which 
form a special class of Fuchsian differential equations which have regular singular points only at three points $0,1, \infty$ and no accessory parameters (rigid system).

We find that defining $G$ by $G(s)=(s-1)^{-L} F(s)$ the differential equations for $G_{j}$ become a special kind of generalised hypergeometric differential equations.

For a given generalised differential equation, there corresponds a system of firstorder differential equations of Okuba type. The explicit relationship is given for example in [5]. However the converse direction seems not to be known. In fact as we will see in our case each $G_{j}$ satisfies different generalised differential equations.

The detailed proof will appear elsewhere.

2. A normal form of differential equations. The differential equations for $G$ takes the following form:

$$
\begin{aligned}
& N \frac{d G}{d s}=\left(-\frac{L}{s-1} A_{1}+\frac{1}{s} A_{0}\right) G, \\
& A_{1}=\left(\begin{array}{ccc}
1 & \cdots & 1 \\
\vdots & \ddots & \vdots \\
1 & \cdots & 1
\end{array}\right), \quad A_{0}=\left(\begin{array}{cccc}
0 & 0 & \cdots & 0 \\
L & -1 & \ddots & \vdots \\
\vdots & \ddots & \ddots & 0 \\
L & \cdots & L & -N+1
\end{array}\right) .
\end{aligned}
$$

First we look for an $N$ th-order matrix $P$ and numbers $a_{j}, b_{j}$ which satisfy the following relations:

$$
\begin{gathered}
\frac{1}{N} L P A_{1}=\left(\begin{array}{ccccc}
0 & 0 & & \cdots & 0 \\
\vdots & \vdots & \ddots & \vdots \\
0 & 0 & \cdots & 0 \\
a_{0} & a_{1}-b_{1} \cdots & a_{N-2}-b_{N-2} & a_{N-1}-b_{N-1}
\end{array}\right) P \\
\frac{1}{N} P A_{0}=\left(\begin{array}{ccccc}
0 & 1 & 0 & \cdots & 0 \\
\vdots & \ddots & \ddots & \ddots & \vdots \\
0 & & \ddots & & 0 \\
0 & -b_{1} & \cdots & -b_{N-2} & -b_{N-1}
\end{array}\right) P
\end{gathered}
$$

If we can find such non-singular matrix $P$, then the first component $(P G)_{1}$ of $P G$ is annhilated by the generalised hypergeometric differential operator with the parameters $a_{j}, b_{j}$ :

$$
s\left(\sum_{j=0}^{N} a_{j} \vartheta^{j}\right)-\sum_{j=1}^{N} b_{j} \vartheta^{j}, \quad \vartheta=s \frac{d}{d s}, \quad a_{N}=1, b_{N}=1 .
$$

Factorising as

$$
\begin{array}{r}
\sum_{j=0}^{N} a_{j} \vartheta^{j}=\prod_{j=1}^{N}\left(\vartheta+\alpha_{j}\right), \\
\sum_{j=1}^{N} b_{j} \vartheta^{j}=\vartheta \prod_{j=1}^{N-1}\left(\vartheta+\beta_{j}-1\right),
\end{array}
$$

we have another form of generalised hypergeometric differential operator. 
3. Transformation matrix. Define

$c_{i}=(r(N, i-1, x)-s(N, i-1)) /(-N)^{N-i+1}, \quad f_{i}=-s(N, i-1) /(-N)^{N-i+1}$,

where $r(N, i, x)$ is defined by

$$
\sum_{i=0}^{N} r(N, i, x) t^{i}=\prod_{j=1}^{N}(t+x-j+1)
$$

and $s(N, i)$ denotes the Stirling number of the first kind.

We set

$$
p_{i j}=(-1)^{N+j} \sum_{s=0}^{j-1}\left(\begin{array}{c}
N-L-1 \\
s
\end{array}\right)\left(\begin{array}{c}
L \\
j-1-s
\end{array}\right)(L+s)^{i-1} /(-N)^{i-1}
$$

and consider the square matrix $P$ of order $N$ with its $(i, j)$ entries $p_{i j}$.

Proposition 1. The matrix P satisfies

$$
\begin{aligned}
\frac{L}{N} P\left(\begin{array}{ccc}
1 & \cdots & 1 \\
\vdots & \ddots & \vdots \\
1 & \cdots & 1
\end{array}\right) & =\left(\begin{array}{ccc}
0 & \cdots & 0 \\
\vdots & & \vdots \\
0 & \cdots & 0 \\
c_{1} & \cdots & c_{N}
\end{array}\right) P, \\
\frac{1}{N} P\left(\begin{array}{ccccc}
0 & 0 & \cdots & 0 \\
L & -1 & \ddots & \vdots \\
\vdots & \ddots & \ddots & 0 \\
L & \cdots & L & -N+1
\end{array}\right) & =\left(\begin{array}{ccccc}
0 & 1 & 0 & \cdots & 0 \\
\vdots & \ddots & \ddots & \ddots & \vdots \\
& & \ddots & & 0 \\
0 & & \cdots & 0 & 1 \\
0 & f_{2} & \cdots & f_{N-1} & f_{N}
\end{array}\right) P .
\end{aligned}
$$

4. Inverse matrix. Define $q_{i j}$ by the relation

$$
\sum_{j=0}^{N-1} q_{i, j+1} t^{j}=\prod_{k=0}^{i-2}(t-L-k) \prod_{k=i}^{N-1}(t-k)
$$

The matrix $Q$ with entries $(-N)^{j-1} q_{i j}$ satisfies the relation

$$
Q P=\prod_{k=1}^{N}(-L+k) I_{N}
$$

where $I_{N}$ is the identity matrix of order $N$.

5. Scalar differential operator. Assume $L>N$. Then using Proposition 1 we see that the entries in (1), (2) are given by

$$
\begin{aligned}
& b_{j}=-(-N)^{-N+j-1} s(N, j-1), \\
& a_{j}=(-N)^{-N+j-1} N^{-N+j-1} r(N, j-1,-L) .
\end{aligned}
$$


The corresponding $N$ th-order differential operator (3) is expressed as

$$
s \prod_{k=1}^{N}\left(\vartheta+\frac{L+k-1}{N}\right)-\prod_{k=1}^{N}\left(\vartheta+\frac{k-1}{N}\right) .
$$

Defining $H=P G$, we see that the first component $H_{1}$ is annihilated by the above operator.

Further using the inverse matrix $Q$ components of $G$ are given as

$$
\begin{aligned}
G_{i} & =\sum_{j=1}^{N}(-N)^{j-1} q_{i j} H_{j} / \prod_{k=1}^{N}(k-L) \\
& =(-1)^{N-1} \prod_{k=0}^{i-2}(N \vartheta+L+k) \prod_{k=i}^{n-1}(N \vartheta+k) H_{1} .
\end{aligned}
$$

Defining

$$
L_{i}=s \prod_{k=1}^{n}(N \vartheta+L+i+k-2)-\prod_{k=1}^{n}(N \vartheta+i-k)
$$

and using

$$
\vartheta s=s(\vartheta+1)
$$

we have the following.

THEOREM 1.

$$
L_{i} G_{i}=0, \quad i=1, \ldots, N .
$$

Rewriting these differential equations those for $F_{j}$ and assuming that $L$ is a positive integer, we proved the conjecture of von Gehlen and Roan.

6. Power series solutions at $s=0$. Here we assume that $L$ is a positive integer. Let us consider generalised hypergeometric series

$$
\begin{aligned}
& F\left(\begin{array}{ccccc}
\alpha_{1}, & \alpha_{2}, & \cdots, & \alpha_{n} & \\
\beta_{1}, & \beta_{2}, & \cdots, & \beta_{n-1}, & 1
\end{array}\right) \\
& =\sum_{k=0}^{\infty} \frac{\left(\alpha_{1}\right)_{k}\left(\alpha_{2}\right)_{k} \cdots\left(\alpha_{n}\right)_{k}}{\left(\beta_{1}\right)_{k}\left(\beta_{2}\right)_{k} \cdots\left(\beta_{n-1}\right)_{k} k !} s^{k}, \\
& (\alpha)_{k}=\alpha(\alpha+1) \cdots(\alpha+k-1),
\end{aligned}
$$

where $\alpha_{1}, \ldots, \alpha_{n}, \beta_{1}, \ldots, \beta_{n-1}$ are parameters. The symbols $(\alpha)_{k}$ are sometimes called Pochhammer symbol.

As is known solutions of generalised hypergeometric differential equation (3) around $s=0$ are given by

$$
F\left(\begin{array}{ccccc}
\alpha_{1}, & \alpha_{2}, & \cdots, & \alpha_{n} \\
\beta_{1}, & \beta_{2}, & \cdots, & \beta_{n-1}, & 1
\end{array} \mid s\right)
$$


with $\alpha_{1}, \ldots, \alpha_{N}, \beta_{1}, \ldots, \beta_{N-1}$ defined by the relations (4), (5) and also

$$
\begin{aligned}
& s^{1-\beta_{j}} F\left(\begin{array}{lll}
1+\alpha_{1}-\beta_{j}, & \cdots, & 1+\alpha_{j-1}-\beta_{j}, \\
1+\beta_{1}-\beta_{j}, & \cdots, & 1+\beta_{j-1}-\beta_{j}
\end{array}\right. \\
& \begin{array}{ccccc}
1+\alpha_{j}-\beta_{j}, & 1+\alpha_{j+1}-\beta_{j}, & \cdots, & 1+\alpha_{N}-\beta_{j} & \\
2-\beta_{j}, & 1+\beta_{j+1}-\beta_{j}, & \cdots, & 1+\beta_{N-1}-\beta_{j} & 1
\end{array}
\end{aligned}
$$

for $j=1, \ldots, N-1$.

Therefore in our case the power series solutions of $L_{i} f=0$ are given by the following generalised hypergeometric series:

$$
F\left(\begin{array}{cccccc}
\frac{L+i-1}{N}, & \frac{L+i}{N}, & \cdots, & \frac{L+N-1}{N}, & \cdots, & \frac{L+i+N-2}{N} \\
\frac{i}{N}, & \frac{i+1}{N}, & \cdots, & 1, & \cdots, & \frac{i+N-1}{N}
\end{array}\right) .
$$

In our case since the parameters are special, the product of Pochhammer symbols in the coefficients are simplified. As a result we have the following series:

$$
\sum_{k=0}^{\infty} \frac{(L+i-1)_{k N}}{(i)_{k N}} s^{k}
$$

We see that these are essentially a sum of binominal series in $s^{1 / N}$ :

$$
\begin{aligned}
& \frac{1}{N} \sum_{j=0}^{N} f_{i}\left(\omega^{j} s^{1 / N}\right), \quad \omega=\exp (2 \pi i / N), \\
f_{i}(x)= & \sum_{n=0}^{\infty} \frac{(L+i-1)_{n}}{(i)_{n}} x^{n} \\
= & \frac{1}{\left(\begin{array}{c}
-L \\
i-1
\end{array}\right)}\left(x^{1-i}(1-x)^{-L}-x^{1-i} \sum_{k=0}^{i-2}\left(\begin{array}{c}
-L \\
k
\end{array}\right)(-x)^{k}\right) .
\end{aligned}
$$

ACKNOWLEDGEMENT. This work was partially supported by JSPS Grant-in-Aid for Scientific Research B-17340046.

\section{REFERENCES}

1. G. Albertini, B. M. McCoy and J. H. H. Perk, Eigenvalue spectrum of the superintegrable chiral Potts model, in Advanced studies in pure mathematics, vol. 19 (Kinokuniya Academic, Tokyo, 1989), 1-55.

2. R. J. Baxter, The superintegrable chiral Potts model, Phys. Lett. A, 133, 185-189.

3. G. von Gehlen, Onsager's algebra and partially orthogonal polynomials, Int. J. Mod. Phys. B, 16 (2002), 2129-2136.

4. G. von Gehlen and S. S. Roan, The superintegrable chiral Potts quantum chain and generalized Chebyshev polynomials, in Integrable structure of exactly solvable two-dimensional models of quantum field theory, vol. 35 (Pakuliak S. and von Gehlen G., Editors) (Kluwer Academic Publisher, Dordrecht, 2001), 155-172.

5. K. Okubo, K. Takano and S. Yoshida, A connection problem for the generalized hypergeometric equation. Funkcial. Ekvac. 31 (1988), 483-495.

6. S. S. Roan, Structure of certain Chebyshev-type polynomials in Onsager's algebra representation, J. Comput. Appl. Math. 202 (2007), 88-1-4. 